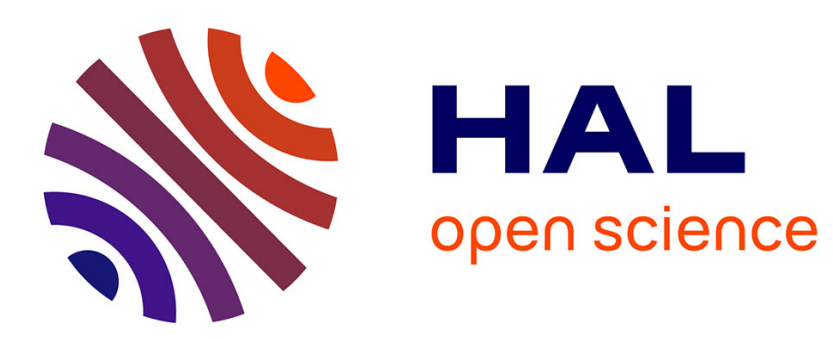

\title{
Buckling of a Short Cylindrical Shell Surrounded by an Elastic Medium
}

\author{
Salah Naili, Christian Oddou
}

\section{To cite this version:}

Salah Naili, Christian Oddou. Buckling of a Short Cylindrical Shell Surrounded by an Elastic Medium. Journal of Applied Mechanics, 2000, 67 (1), 10.1115/1.321173 . hal-01870695

\section{HAL Id: hal-01870695 \\ https://hal.science/hal-01870695}

Submitted on 8 Sep 2018

HAL is a multi-disciplinary open access archive for the deposit and dissemination of scientific research documents, whether they are published or not. The documents may come from teaching and research institutions in France or abroad, or from public or private research centers.
L'archive ouverte pluridisciplinaire HAL, est destinée au dépôt et à la diffusion de documents scientifiques de niveau recherche, publiés ou non, émanant des établissements d'enseignement et de recherche français ou étrangers, des laboratoires publics ou privés. 


\section{Buckling of a Short Cylindrical Shell Surrounded by an Elastic Medium}

\author{
S. Naili \\ e-mail: naili@univ-paris12.fr

\section{Oddou} \\ e-mail: oddou@univ-paris12.fr \\ Laboratoire de Mécanique Physique, UPRES-A CNRS \\ 7052, Université Paris XII, Val de Marne, Faculté \\ des Sciences et Technologie, 61, avenue du Général de \\ Gaulle, 94010 Créteil Cedex, France
}

The lateral surface of a cylindrical structure, which is composed of a thin tube embedded in a large outer medium, is submitted to a uniform external pressure. The buckling pressure of such a structure, corresponding to a low flexural state of the inner tube wall, is theoretically analyzed on the basis of the asymptotic method. The theoretical results are compared with experimental ones obtained from a compression test realized on an elastic tube inserted in a foam. It is found that the Euler pressure and the associated buckling mode index strongly depend upon the rheological and geometrical parameters of both the tube and the surrounding medium. [S0021-8936(00)00201-4]

\section{Formulation of the Problem and Buckling Study}

A nonhomogeneous cylindrical structure composed of a thin shell inserted in a surrounding elastic medium was subjected to a state of plane strain by external pressurization and zero axial longitudinal displacement constraint. The onset of the buckling process for such a structure was analyzed. The theoretical results were compared with original experimental ones as derived from a hoop compression test which was conducted with elastic rubber tubes embedded in foamy materials.

Thus, we consider the mechanical behavior of a cylindrical nonhomogeneous structure made of an internal shell confined in a large outer medium, the whole structure being submitted to a uniform pressure $p$ on its external lateral surface. Each solid is elastic, cylindrical - of same axis - with a circular cross section in the reference configuration. In this configuration, the mean radius of the shell is denoted as $r_{0}$. The outer radius $r_{\infty}$ of the medium is assumed to be very large compared to $r_{0}$. We will denote as $e_{0}$ the thickness of the shell. The two solids have the same height which is small in comparison with $r_{\infty}$. The outer lateral boundary of the medium is subjected to a uniform pressure $p$. We assume frictionless contact between the two tubes. Body forces and inertia effects are deemed to be negligibly small.

The work of Forrestal and Herrman [1] presents solutions for both bonded and smooth conditions at the shell-medium interface; it includes a geometrically nonlinear formulation for both the shell and the medium. Moore and Booker [2] presented the linear approximation of Forrestal and Herrman's formulation. The physical unknowns which are involved in these theoretical formulations are the buckling pressure of the shell in the first work and the hoop compression stress inside this shell in the second one.

In the present work, we use the dimensionless buckling pressure of the overall structure consisting of the shell and surrounding medium developed by Razakamiadana et al. [3] and which is given by the relation

$$
\bar{p}=n^{2}-1+\frac{\bar{\beta}}{n^{2}-1},
$$

where the dimensionless variables are given as follows:

$$
\bar{p}=\alpha p \frac{r_{0}^{3}}{D}, \quad \bar{\beta}=\beta \frac{r_{0}^{4}}{d},
$$

and where $D=E_{1} e_{0}^{3} / 12\left(1-\nu_{1}^{2}\right)$ is the flexural rigidity modulus of the shell, $E_{1}$ and $\nu_{1}$ being, respectively, its Young's modulus and its Poisson's ratio, while the index $n$ characterizes the buckling mode. The parameters $\alpha$ and $\beta$ are defined by

$$
\begin{gathered}
\alpha=\frac{\left[\frac{\lambda_{2}+2 \mu_{2}}{\lambda_{2}+\mu_{2}}\right]\left(2 \frac{e_{0}}{r_{0}}\right)}{1-\left(1-2 \frac{e_{0}}{r_{0}}\right)\left[1-\frac{\mu_{2}}{\mu_{1}}\right]+\frac{\mu_{2}}{\lambda_{1}+\mu_{1}}}, \\
\beta=2 \frac{\mu_{2}}{r_{0}}\left(\lambda_{2}+\mu_{2}\right)\left[\frac{n^{2}-1}{n\left(\lambda_{2}+2 \mu_{2}\right)+\mu_{2}}\right],
\end{gathered}
$$

in which the Lame's parameters of the shell and of the medium are denoted by $\lambda_{1}, \mu_{1}$ and $\lambda_{2}, \mu_{2}$, respectively.

In this study, we were interested in the smallest value of the buckling pressure - the Euler pressure-, while varying the index $n$ associated with the buckling mode; such a mode index characterizes the number of axes of symmetry in the actual configuration. Indeed, this minimal pressure is the most frequently observed experimentally while applying incremental loading to the structure. This pressure is expressed as

$$
p_{e}=\min _{n \geqslant 2}\left[\frac{1}{\alpha}\left(\left(n^{2}-1\right) \frac{D}{r_{0}^{3}}+\frac{\beta r_{0}}{n^{2}-1}\right)\right] .
$$

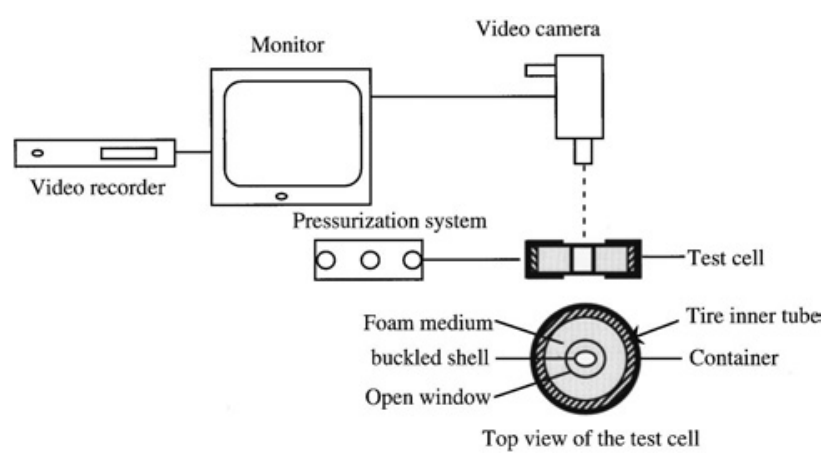

Fig. 1 Experimental apparatus for hoop compression tests. The tube inserted in the foam medium is in a buckled state with index of buckling mode equal to two. 
Table 1 Experimental and theoretical results of Euler pressure normalized by $E_{2}$ and index of buckling mode $n$ associated for various dimensionless mechanical and geometrical parameters

\begin{tabular}{|c|c|c|c|c|c|c|c|}
\hline \multicolumn{6}{|c|}{ Experiment } & \multicolumn{2}{|c|}{ Theory } \\
\hline $\bar{E}$ & $E_{2}(\mathrm{kPa})$ & $\bar{e}$ & $e_{0}(\mathrm{~mm})$ & $p_{e} / E_{2} \times 10^{-2}$ & $n$ & $p_{e} / E_{2} \times 10^{-2}$ & $n$ \\
\hline 14.50 & 100 & 0.078 & 1.00 & $(8.69 \pm 0.80)$ & 6 & 8.39 & 6 \\
\hline 14.50 & 100 & 0.189 & 2.40 & $(15.34 \pm 2.19)$ & 2 & 13.00 & 2 \\
\hline 26.07 & 79 & 0.037 & 0.46 & $(4.21 \pm 0.40)$ & 4 & 4.21 & 6 \\
\hline 28.70 & 100 & 0.105 & 1.30 & $(11.00 \pm 1.00)$ & 4 & 9.80 & 4 \\
\hline 28.70 & 100 & 0.136 & 1.75 & $(12.89 \pm 0.92)$ & 3 & 12.05 & 3 \\
\hline 28.86 & 79 & 0.032 & 0.40 & $(3.98 \pm 0.33)$ & 3 & 4.87 & 6 \\
\hline 28.86 & 79 & 0.070 & 0.90 & $(6.13 \pm 0.63)$ & 2 & 7.65 & 5 \\
\hline 29.85 & 69 & 0.037 & 0.46 & $(4.66 \pm 0.78)$ & 4 & 5.24 & 6 \\
\hline 33.00 & 69 & 0.032 & 0.40 & $(4.21 \pm 0.21)$ & 3 & 4.79 & 6 \\
\hline 33.00 & 69 & 0.070 & 0.90 & $(6.10 \pm 0.31)$ & 2 & 7.69 & 5 \\
\hline 158.46 & 13 & 0.037 & 0.46 & $(11.76 \pm 3.69)$ & 3 & 7.60 & 3 \\
\hline 175.38 & 13 & 0.070 & 0.90 & $(17.69 \pm 0.10)$ & 2 & 9.61 & 2 \\
\hline
\end{tabular}

It is to be noted here that a classical case corresponds to the particular condition of an external incompressible fluid-i.e., $\mu_{2}$ $=0$ and $\lambda_{2} \rightarrow \infty$-surrounding the shell, so that $\alpha=1$ and $\beta=0$ in relations (1) and (2).

\section{Experimental Procedure}

Hoop compression tests were performed on a cylindrical structure with a circular cross section composed by a thin rubber shell, of external radius $r_{e}=13 \mathrm{~mm}$ which was inserted in a large foam medium of external radius $r_{\infty}=110 \mathrm{~mm}$. Both tubes had a height $H=30 \mathrm{~mm}$. The thin rubber shell was slightly stressed when inserted within the foam medium in order to establish a good contact between the two solids.

A steady loading was applied on the external lateral wall of the surrounding foam medium by means of a tire inner tube connected to a standard pressurization system. The plane strain of the structure was obtained by maintaining it between two circular and polished PMMA transparent plates. In order to avoid significant friction between the foam and the plates, the lower and upper faces of the foam were sprinkled with talc powder.

The applied pressure was measured by using a mercury $U$-manometer graded every $1 \mathrm{~mm}$ in height with a maximum reading error estimated at about $0.5 \mathrm{~mm}$. When the buckling pressure is "very low," the relative accuracy of the pressure measurement was estimated at about seven percent. But, in 90 percent of the cases, the measured pressure was about $50 \mathrm{~mm} \mathrm{Hg}$ and the relative uncertainty of measurement was estimated, on average, to be one percent.

With this experimental setup, several tests of compression were conducted on the structure with given geometrical and mechanical characteristics. The tested structure was submitted to a gradual and slow loading so that, for each step, the system can be considered in stationary equilibrium state. The shape of the cross section of the shell remains circular before undergoing a change of shape. We monitored the evolution of shape with a CCD camera video placed on the axis of the tubes-see Fig. 1.

The thickness $e_{0}$ of the shell, in its reference configuration, was inferred from the mean value of the measurements conducted with a micrometer at various locations on the wall. The variations around the mean value were found to be in the order of two percent. The values of Young's modulus of the shell and of the medium were derived from traction and compression tests, respectively applied on samples of the constitutive materials. In the different experimental setup, four types of latex foam media with different Young's modulus and Poisson's ratio were combined with tubes made of various PCP, PCV, or latex materials having different characteristic mechanical properties as indicated in Table 1. For the deformations up to ten percent each Young's modulus was evaluated with a maximum error of five percent. Besides, these tests have shown that the Poisson's ratio of the shell was about 0.5-, i.e., the material is incompressible-whereas the Poisson's ratio for the foam media were around zero.

Next, the video images were digitized and then automatically processed using a global thresholding method so as to quantify the inner cross section area of the inserted tube and to characterize its shape. In the extreme case corresponding to a significant variation of this shape, the relative uncertainty of area measurement was estimated to be of the order of two percent. Indeed, when the structure is submitted to a gradual and slow loading, we retained as Euler pressure $p_{e}$, the one which corresponds to a clear change in the inner cross section area, as discussed later on.

For a given structure, the measurement of the buckling pressure was repeated ten times at least and the relative gap compared to the mean value varies between 1 percent and 30 percent.

\section{Analysis and Discussion}

We show, in columns 5 and 6 of Table 1, the experimental results obtained on 110 tests implying 12 structures of different geometry and elastic properties. The results are discussed by using the dimensionless geometrical and mechanical parameters $\bar{e}$ $=e_{0} / r_{0}$ and $\bar{E}=E_{1} / E_{2}$ in the case of rather thin tubes and exter-

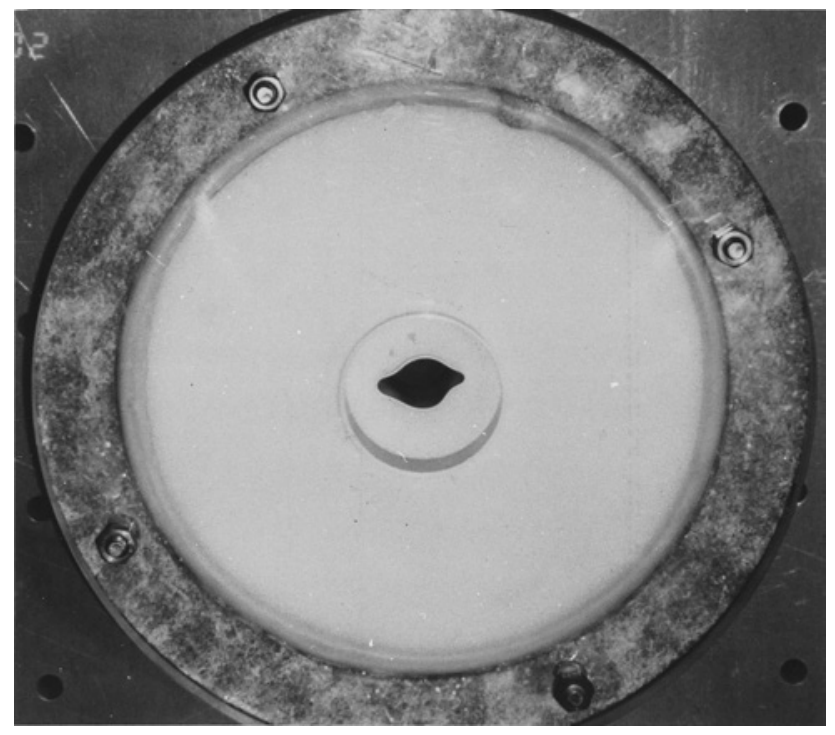

Fig. 2 Top view of the test cell giving an illustrative example of the tube inserted in the foam medium in a buckled state with index of buckling mode equal to four. Circular windows-with a radius in the order $30 \mathrm{~mm}$-were cut on the top and bottom of the PPMA container for a better definition of the image during the recording. 
nal foamy material softer than the rubbery one of the tube. Starting with Eq. (2), we determined the variations of the Euler pressure $p_{e}$ normalized by $E_{2}$-denoted as $\bar{p}_{e}$-as a function of $\bar{E}$ for various values of $\bar{e}$. The associated buckling mode index $n$ then depends on $\bar{E}$. It is worth noting that the assessment of the buckling pressure in such an experiment was based on the variation of a global geometrical parameter such as the area of the internal cross section of the inserted shell-see Fig. 2. Other more sensitive parameters, related to the changes in local shape properties could, however, be envisaged but their quantification by an image-processing system would have been more difficult to implement.

In columns 7 and 8 of Table 1 , the theoretical results are compared with experimental ones. These results show that the Euler pressures, evaluated theoretically and determined experimentally, agree well accounting for the inherent scatter in experimental measurements. Moreover, the mode index $n$ associated with $\bar{p}_{e}$ coincides exactly for 50 percent of the cases. Nevertheless, it is worth emphasizing that significant differences arised in the case of very thin tubes for which the mode index are rather high, the large number of lobes being more sensitive to small heterogeneities in material property and geometry. Despite this, the observed experimental results are, in general, well reproduced by our theoretical model.

\section{References}

[1] Forrestal, M. J., and Herrmann, G., 1965, "Buckling of a Long Cylinder Shell Surrounded by an Elastic Medium,'” Int. J. Solids Struct., 1, pp. 297-309.

[2] Moore, I. D., and Booker, J. R., 1985, "'Simplified Theory for the Behavior of Buried Flexible Cylinders Under the Influence of Uniform Hoop Compression,' Int. J. Solids Struct., 21, No. 9, pp. 929-941.

[3] Razakamiadana, A., Naili, S., and Oddou, C., 1997, 'Flambement d'une Coque Mince Confinée : Théorie et Expérience,' C. R. Acad. Sci., Ser. Ilb: Mec., Phys., Chim., Sci. Astron., 325, pp. 119-126. 